\title{
Role of Quality Circles \& Total Quality Management Practices in an Indian Public Sector Industry: A Pilot Research
}

\author{
Gopi S, Asher John Sathya, Abhinav Suresh
}

\begin{abstract}
Quality Circle consists of a group of people, who are doing the same or similar work, who meet voluntarily and regularly to identify, analyse, discuss and solve problems in their work areas. Housing Accessory Cover (Rh) casting used in a critical helicopter component which is a magnesium alloy, thin walled casting needs special attention and careful control of all parameters. This casting has several contours with very close dimensional tolerance. Supply of housing accessory $(R h)$ casting for a critical helicopter component was delayed beyond the committed date due to high rejection $(56 \%)$ at floor inspection \& radiography. The goal of this pilot study was to examine the factors influencing the successful implementation of active Quality Circles to increase organizational productivity and establish processes to manufacture zero defect components. One such unit quality circles strives to achieve $100 \%$ radiographic quality housing accessory cover $(\mathrm{Rh})$ casting with zero level of rejection. In a trial run of 200 units, there was a saving of Rs 19,525 on each casting.
\end{abstract}

Index Terms- Quality Circles, Housing Accessory, Zero defect.

\section{INTRODUCTION}

Quality Circle is a small group of people in the same work area who meet voluntarily on a regular basis to identify, analyze, discuss and solve various types of problems in that area, leading to improvement in their performance and enrichment of their work life. Members of a particular Quality Circle should be from the same work area so that problems selected will be familiar to all members. The ideal size of a circle is between 5 and 8 and the size should be such that the circle is effective and should offer enough time to every member to participate and contribute at each meeting. The membership is strictly voluntary. Members are neither compelled to join nor kept out. A Quality Circle process is made up of interrelated parts: the members, the facilitators, the coordinators and the steering committee. The Quality Circle is the basic force in solving work related problems. Some of the Quality Circle's main objectives include ensuring harmony, effective teamwork, job satisfaction, quality improvement, better communication, productivity and team building. The key factors for the success of any Quality Circle is its structure of the functional rapport between its different elements.

\section{LITERATURE REVIEW}

Total quality management is an important and prominent approach to management.[1]The approach and analysis of the problems by means of the quality circle have led to an optimization of the human and financial effort involved.[2][3][4] However, there is still a need for continuous improvement even among organisations recognized for their TQM achievements.[5] Companies perceive benefit from quality Circles in three areas: improvement of work performance, company competitiveness and profitability, and employee outcomes.[6][7][8] The relationship between TQM practice and organisational performance is significant in a cross-sectional point of view.[9][10] Employee performance and innovation performance partially mediate the relationship between TQM practices and firm performance.[11] However, these links are complex.[12][13][14][15] While features such as quality training, process improvement, and benchmarking do not generally produce any advantages, certain tacit, behavioural, imperfectly imitable features such as open culture, employee empowerment, and executive commitment can be significantly advantageous.[16] Studies have shown that total quality management is positively related to manufacturing performance.[17] Quality Improvement Methods with Quality Circles have shown the most significant long-term benefits in terms of increase in sales.[18] The implementation of TQM practices in R\&D divisions and in knowledge management has made significant contribution to $R \& D$ performance of companies[19] as well as their knowledge management.[20]

\section{PILOT STUDY}

\subsection{Identification \& selection of Problems}

In the pilot study conducted a total of 80 problems were identified \& were categorized into three groups

Group A: Problems requiring minimal involvement of other departments.

Group B: Problems requiring involvement of other departments.

Group C: Problems requiring management sanction for implementation. 


\section{ROLE OF QUALITY CIRCLES \& TOTAL QUALITY MANAGEMENT PRACTICES IN AN INDIAN PUBLIC SECTOR INDUSTRY: A PILOT RESEARCH}

Number of problems under Group A, B and C were 46, 23 \& 11 respectively. Selection of problem was done based on the ratings provided by quality circle members to the questionnaire. 'High rejection of housing accessory cover castings' was rated as the most critical problem as per the responses received.

\subsubsection{Problem Statement}

Housing accessory cover (Rh) Casting is used in a critical helicopter component. This is a magnesium alloy formed by thin walled casting. It needs special attention and careful control of all parameters casting has several contours with very close dimensional tolerance. Supply of housing Accessory Cover (Rh) casting for a critical helicopter component project was delayed beyond the committed date due to high rejection at floor inspection and radiography. The existing rejection rate at the time of conducting the study was $56 \%$.

\subsection{Data Analysis of problem}

Brain storming sessions among various quality circles identified the reasons for rejections, which caused the supply of housing accessory cover casting of the project beyond the committed dates were;

a) Shrinkage: Change in volume of metal as it changes from liquid to solid phase.

b) Micro Shrinkage: Very finely divided porosity resulting from interdendritic shrinkage.

c) Inclusion: Particles of impurities, usually Oxides, Sulphides and Silicates.

d) Non-Formation: Non-fusion of two metal streams in the casting.

Table 1: Rejection Data Analysis.

\begin{tabular}{|c|c|c|c|}
\hline Sl.no. & Melt No. & Place of Rejection & $\begin{array}{l}\text { Reason for } \\
\text { Rejection }\end{array}$ \\
\hline 1 & MG 02 AP 06 & $\begin{array}{l}\text { Floor, Radiography } \\
\text { Inspection }\end{array}$ & $\begin{array}{l}\text { Shrinkage, Non - } \\
\text { Formation }\end{array}$ \\
\hline 2 & M5 02 AP 15 & Radiography Inspection & $\begin{array}{c}\text { Micro Shrinkage, } \\
\text { Shrinkage }\end{array}$ \\
\hline 3 & MG 02 AP 17 & Radiography Inspection & $\begin{array}{l}\text { Micro Shrinkage, } \\
\text { Shrinkage, Inclusion }\end{array}$ \\
\hline 4 & MG 02 JU 08 & Radiography Inspection & $\begin{array}{c}\text { Micro Shrinkage, } \\
\text { Shrinkage, Inclusion }\end{array}$ \\
\hline 5 & MG 02 NR 40 & $\begin{array}{l}\text { Floor, Radiography } \\
\text { Inspection }\end{array}$ & $\begin{array}{c}\text { Non - Formation, } \\
\text { Shrinkage }\end{array}$ \\
\hline 6 & MG 02 DR 05 & Radiography Inspection & $\begin{array}{c}\text { Micro Shrinkage, } \\
\text { Shrinkage }\end{array}$ \\
\hline 7 & MG 02 JU 18 & $\begin{array}{l}\text { Floor, Radiography } \\
\text { Inspection }\end{array}$ & Non - Formation \\
\hline 8 & MG 02 JU 24 & Radiography Inspection & $\begin{array}{l}\text { Micro Shrinkage, } \\
\text { Inclusion }\end{array}$ \\
\hline 9 & MG 02 JU 33 & Radiography Inspection & $\begin{array}{c}\text { Micro Shrinkage, } \\
\text { Shrinkage }\end{array}$ \\
\hline 10 & MG 02 JU 40 & Radiography Inspection & Shrinkage \\
\hline
\end{tabular}

Observation: 10 batches of housing accessory cover $(\mathrm{Rh})$ were rejected during floor inspection \& Radiography due to defects.

\subsubsection{Casting Specifications}

Table 2: Casting specifications

\begin{tabular}{ll}
\hline Project & Critical helicopter component \\
Part number & 1201p 636h 211 702 \\
Part Name & Housing accessory cover \\
Material & Rz5 (Mg alloy) \\
Unit Weight & $10 \mathrm{Kg}$ \\
Casting type & Sand casting \\
Value per casting & Rs 19,525 \\
Gating Ratio & $2.8: 3.6: 1$ \\
Pouring Temperature & $750^{\circ} \mathrm{C}$ \\
\hline
\end{tabular}

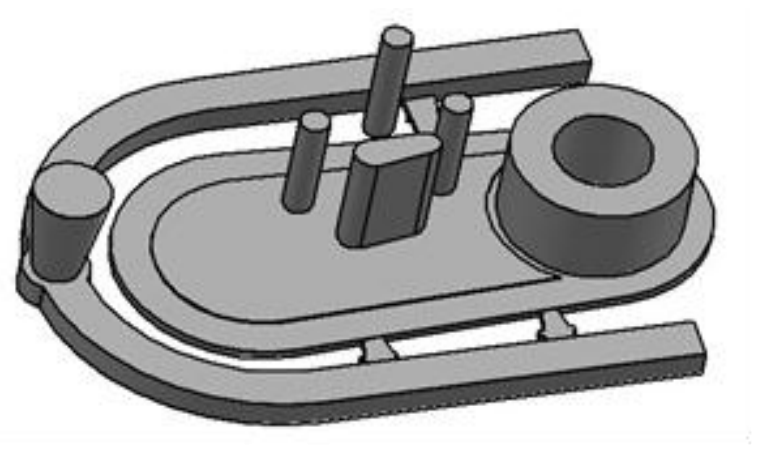

Fig-1: Housing accessory cover

\subsubsection{Identification of Root Causes}

During Brainstorming session, the members followed a systematic approach to identify and analyse the causes for rejections in Housing Accessory Cover (Rh) Casting.

\subsubsection{Shrinkage and Micro-Shrinkage}

1. Insufficient risers

2. High pouring temperatures

3. Absence of chills

4. Fine sand for facing

5. Improper calibration of Pyrometer

6. Absence of heat insulating sleeves

7. Absence of blind risers

8. Lack of concentration

9. Lack of skill

10. Overheating of Mould \& Cores

11. Improper mixing of molten metal

\subsubsection{Non - Formation}

1. Inaccurate Pyrometer

2. Inappropriate gating ratio

3. Change of location of Ingates

4. Poor surface finish of pattern

5. Improper skimming of molten metal

6. Non-uniform preheating of Molds \& Cores

7. Improper calibration of Pyrometer

8. Low pouring temperature

9. Lack of concentration

10. Lack of skill

\subsubsection{Inclusion}


1. Inappropriate mixing of sand and binder

2. Inappropriate AFS sand size used

3. Improper filtering system

4. Problem with mould coat

5. Faulty skimmer \& cleaner

6. Absence of Flow Offs

7. High pouring temperature

8. Poor quality binder

9. Improper Sulphur spray

10. Inadequate skimming of liquid metal

\subsubsection{Root Cause Analysis}

\subsubsection{Identification by Validation}

Table- 3. Defect: Shrinkage and Micro Shrinkage

\begin{tabular}{|c|c|c|c|c|}
\hline Sl.no. & Cause & Observation & Verification & Conclusion \\
\hline 1 & $\begin{array}{l}\text { Insufficient } \\
\text { Risers }\end{array}$ & $\begin{array}{l}\text { Absence of } \\
\text { Riser }\end{array}$ & $\begin{array}{c}\text { Riser } \\
\text { introduced in } \\
\text { Trial-1 Melt } \\
\text { no. } \\
\text { MG04JU18 } \\
\text { avoids } \\
\text { shrinkage }\end{array}$ & Cause \\
\hline 2 & $\begin{array}{l}\text { Absence of Heat } \\
\text { Insulating } \\
\text { Sleeves }\end{array}$ & $\begin{array}{l}\text { No need of } \\
\text { sleeves }\end{array}$ & $\begin{array}{c}\text { As per Hps } \\
625.2\end{array}$ & Not a cause \\
\hline 3 & $\begin{array}{l}\text { Overheating of } \\
\text { Molds \& Cores }\end{array}$ & $\begin{array}{c}\text { Preheating } \\
\text { Temperature } \\
=110^{\circ} \mathrm{C}\end{array}$ & $\begin{array}{l}\text { Contact Tip } \\
\text { Pyrometer }\end{array}$ & Not a cause \\
\hline 4 & $\begin{array}{l}\text { Absence of } \\
\text { Blind Risers }\end{array}$ & $\begin{array}{l}\text { No need of } \\
\text { Blind Risers }\end{array}$ & $\begin{array}{c}\text { As per Hps } \\
612.3\end{array}$ & Not a cause \\
\hline 5 & $\begin{array}{l}\text { Improper mixing } \\
\text { of Molten Metal }\end{array}$ & $\begin{array}{l}\text { Standard } \\
\text { Practice }\end{array}$ & $\begin{array}{c}\text { As per Hps } \\
612.3\end{array}$ & Not a cause \\
\hline 6 & $\begin{array}{l}\text { High pouring } \\
\text { temperature }\end{array}$ & $\begin{array}{c}\text { Pouring } \\
\text { Temperature } \\
=750^{\circ} \mathrm{C}\end{array}$ & $\begin{array}{c}\text { Trial-1\&2 } \\
\text { Melt no. } \\
\text { MG04JU18 } \\
\& \\
\text { MG04JU24 } \\
\text { Pouring } \\
\text { Temperature } \\
=730^{\circ} \mathrm{c}\end{array}$ & Cause \\
\hline 7 & $\begin{array}{l}\text { Absence of } \\
\text { Chills }\end{array}$ & $\begin{array}{l}\text { No Chills } \\
\text { used for } \\
\text { mould } \\
\text { preparation }\end{array}$ & $\begin{array}{c}\text { Chills used in } \\
\text { Trial-1\&2 } \\
\text { Melt no. } \\
\text { MG04JU18 } \\
\& \\
\text { MG04JU24 } \\
\text { avoids micro } \\
\text { shrinkage }\end{array}$ & Cause \\
\hline 8 & $\begin{array}{c}\text { Improper } \\
\text { calibration of } \\
\text { Pyrometer }\end{array}$ & $\begin{array}{l}\text { Pyrometer } \\
\text { calibrated }\end{array}$ & $\begin{array}{c}\text { Calibrate as } \\
\text { per Fdy - } \\
2340\end{array}$ & Not a cause \\
\hline 9 & Lack of skill & $\begin{array}{l}\text { Skilled } \\
\text { moulders }\end{array}$ & $\begin{array}{l}\text { Certification } \\
\text { of Skills }\end{array}$ & Not a cause \\
\hline 10 & $\begin{array}{c}\text { Lack of } \\
\text { concentration }\end{array}$ & $\begin{array}{l}\text { Dedicated } \\
\text { moulders }\end{array}$ & $\begin{array}{l}\text { Certification } \\
\text { of Dedication }\end{array}$ & Not a cause \\
\hline 11 & $\begin{array}{l}\text { Fine sand for } \\
\text { Facing }\end{array}$ & $\begin{array}{c}\text { Silica Sand } \\
\text { AFS } 60 \text { used }\end{array}$ & $\begin{array}{c}\text { Zircon AFC } \\
80 \text { used in } \\
\text { Trial-2 Melt } \\
\text { no. } \\
\text { MG04JU24 } \\
\text { avoids micro } \\
\text { shrinkage }\end{array}$ & Cause \\
\hline
\end{tabular}

Table-4. Defect: Non - Formation.

\begin{tabular}{|c|c|c|c|c|}
\hline Sl.no. & Cause & Observation & Verification & Conclusion \\
\hline 1 & $\begin{array}{l}\text { Inaccurate } \\
\text { Pyrometer }\end{array}$ & $\begin{array}{l}\text { Usage of } \\
\text { calibrated } \\
\text { Pyrometer }\end{array}$ & $\begin{array}{l}\text { Calibrated as } \\
\text { per Fdy-5667 }\end{array}$ & Not a cause \\
\hline 2 & $\begin{array}{l}\text { Inappropriate } \\
\text { Gating Ratio }\end{array}$ & $\begin{array}{c}\text { Gating ratio } \\
3.7: 4.7: 1\end{array}$ & $\begin{array}{l}\text { As per Trial-3 } \\
\text { Melt no. } \\
\text { MG04JU33 with } \\
\text { modified gating } \\
\text { ratio 2.8: } 3.6: 1\end{array}$ & Cause \\
\hline 3 & $\begin{array}{l}\text { Change the } \\
\text { location of } \\
\text { Ingates }\end{array}$ & $\begin{array}{l}\text { Position of } \\
\text { ingates as per } \\
\text { method } \\
\text { sketch }\end{array}$ & $\begin{array}{c}\text { As per Trial-3 Melt } \\
\text { no. MG04JU33 } \\
\text { with Ingates } \\
\text { Non-Formation } \\
\text { was avoided }\end{array}$ & Cause \\
\hline 4 & $\begin{array}{l}\text { Poor surface } \\
\text { finish of } \\
\text { Pattern }\end{array}$ & $\begin{array}{c}\text { Mould } \\
\text { surface } \\
\text { found } \\
\text { satisfactory }\end{array}$ & $\begin{array}{l}\text { Mould surface } \\
\text { finish as per } \\
\text { Fdy-T-2040 }\end{array}$ & Not a cause \\
\hline 5 & $\begin{array}{c}\text { Improper } \\
\text { skimming of } \\
\text { Molten metal }\end{array}$ & $\begin{array}{l}\text { Mould } \\
\text { surface } \\
\text { found } \\
\text { satisfactory }\end{array}$ & $\begin{array}{c}\text { As per Hps } \\
612.3\end{array}$ & Not a cause \\
\hline 6 & $\begin{array}{l}\text { Non-uniform } \\
\text { Mould \& Core } \\
\text { preheating } \\
\text { temperature }\end{array}$ & $\begin{array}{c}\text { Range of } \\
80^{\circ} \mathrm{C} \text { to } \\
100^{\circ} \mathrm{C}\end{array}$ & $\begin{array}{l}\text { Monitoring of } \\
\text { temperature by } \\
\text { Pyrometer to } \\
\text { required } \\
\text { temperature }\end{array}$ & Not a cause \\
\hline 7 & $\begin{array}{l}\text { Low pouring } \\
\text { temperature }\end{array}$ & $\begin{array}{l}\text { Pouring } \\
\text { temperature } \\
=750^{\circ} \mathrm{C}\end{array}$ & $\begin{array}{c}\text { Trial-3 Melt no. } \\
\text { MG04JU33 } \\
\text { pouring } \\
\text { temperature } \\
730^{\circ} \mathrm{C} \text { not } \\
\text { effected to } \\
\text { Non-Formation }\end{array}$ & Not a cause \\
\hline 8 & $\begin{array}{l}\text { Improper } \\
\text { mould \& core } \\
\text { assembly }\end{array}$ & $\begin{array}{l}\text { As per } \\
\text { standard } \\
\text { practice }\end{array}$ & $\begin{array}{c}\text { As per Hps } \\
615.2\end{array}$ & Not a cause \\
\hline 9 & Lack of Skill & $\begin{array}{l}\text { Skilled } \\
\text { moulders }\end{array}$ & $\begin{array}{l}\text { Certification of } \\
\text { Skills }\end{array}$ & Not a cause \\
\hline 10 & $\begin{array}{c}\text { Lack of } \\
\text { concentration }\end{array}$ & $\begin{array}{l}\text { Dedicated } \\
\text { Moulders }\end{array}$ & $\begin{array}{c}\text { Certification of } \\
\text { dedication }\end{array}$ & Not a cause \\
\hline 11 & $\begin{array}{c}\text { Improper } \\
\text { calibration of } \\
\text { Pyrometer }\end{array}$ & $\begin{array}{c}\text { Skilled } \\
\text { calibration } \\
\text { personnel }\end{array}$ & $\begin{array}{l}\text { Certification of } \\
\text { Skills }\end{array}$ & Not a cause \\
\hline
\end{tabular}


Table -5. Defect: Inclusions.

\begin{tabular}{|c|c|c|c|c|}
\hline Sl.no. & Cause & Observation & Verification & Conclusion \\
\hline 1 & $\begin{array}{l}\text { Inappropriate } \\
\text { mixing of } \\
\text { Sand \& Binder }\end{array}$ & $\begin{array}{l}\text { Standard } \\
\text { practice }\end{array}$ & $\begin{array}{c}\text { Conforms to } \\
\text { Hps } 613.1\end{array}$ & Not a cause \\
\hline 2 & $\begin{array}{l}\text { Improper } \\
\text { filtering } \\
\text { system }\end{array}$ & $\begin{array}{c}\text { Mesh } 16 \times 22 \\
\text { was used }\end{array}$ & $\begin{array}{c}\text { Usage of } 3 \times 2 \times 9 \\
\text { multilayered } \\
\text { mesh Trail-4 } \\
\text { M04JU40 } \\
\text { avoids inclusion }\end{array}$ & Not a cause \\
\hline 3 & $\begin{array}{l}\text { Problem with } \\
\text { mould coat }\end{array}$ & $\begin{array}{c}\text { Proprietary } \\
\text { product }\end{array}$ & $\begin{array}{l}\text { Conforms to } \\
\text { HPS } 610.3\end{array}$ & Not a cause \\
\hline 4 & $\begin{array}{l}\text { Inappropriate } \\
\text { AFC size used }\end{array}$ & AFC $30-35$ & $\begin{array}{c}\text { As per Hps } \\
611.2\end{array}$ & Not a cause \\
\hline 5 & $\begin{array}{c}\text { Faulty } \\
\text { Skimmer \& } \\
\text { Cleaner }\end{array}$ & $\begin{array}{l}\text { Standard } \\
\text { tools }\end{array}$ & $\begin{array}{c}\text { As per } \\
\text { Fdy-1345 }\end{array}$ & Not a cause \\
\hline 6 & $\begin{array}{l}\text { Obscene of } \\
\text { Flow Offs }\end{array}$ & $\begin{array}{c}\text { No Blow offs } \\
\text { at the Runner } \\
\text { bar as per } \\
\text { methods }\end{array}$ & $\begin{array}{l}\text { Introduction of } \\
\text { Flow Offs } \\
\text { avoids } \\
\text { inclusions }\end{array}$ & Not a cause \\
\hline 7 & $\begin{array}{l}\text { High pouring } \\
\text { temperature }\end{array}$ & $\begin{array}{l}\text { Pouring } \\
\text { temperature } \\
=730^{\circ} \mathrm{C}\end{array}$ & $\begin{array}{c}\text { Trial-4 Melt no. } \\
\text { MG04JU40 } \\
\text { pouring } \\
\text { temperature = } \\
730^{\circ} \mathrm{C}\end{array}$ & Not a cause \\
\hline 8 & $\begin{array}{l}\text { Poor quality } \\
\text { Binder }\end{array}$ & $\begin{array}{c}\text { Proprietary } \\
\text { product }\end{array}$ & $\begin{array}{l}\text { Conforms to } \\
\text { Hps } 613.1\end{array}$ & Not a cause \\
\hline 9 & $\begin{array}{c}\text { Improper } \\
\text { Sulphur spray }\end{array}$ & $\begin{array}{l}\text { Standard } \\
\text { practice }\end{array}$ & $\begin{array}{c}\text { As per Hps } \\
612.3\end{array}$ & Not a cause \\
\hline 10 & $\begin{array}{l}\text { Inadequate } \\
\text { skimming of } \\
\text { molten metal }\end{array}$ & $\begin{array}{l}\text { Standard } \\
\text { practice }\end{array}$ & $\begin{array}{c}\text { As per Hps } \\
614.2\end{array}$ & Not a cause \\
\hline
\end{tabular}

\subsubsection{Root Causes}

3.2.4.1 Root Causes of Defect: Shrinkage \& Micro Shrinkage

1. Insufficient risers

2. High pouring temperature

3. Improper directional solidification

\subsubsection{Root Causes of Defect: Non - Formation}

1. Inappropriate Gating Ratio

2. Wrong position of ingates

\subsubsection{Root Causes of Defect: Inclusion}

1. Absence of filters of required specification in the required location

2. Absence of flow offs of required specification in the required location
Table-6. Defect check sheet (Batch Quantity: 2 Nos).

\begin{tabular}{ccccc}
\hline Defect & $\begin{array}{c}\text { Shrinkag } \\
\mathbf{e}\end{array}$ & $\begin{array}{c}\text { Micro } \\
\text { Shrinkag } \\
\mathbf{e}\end{array}$ & $\begin{array}{c}\text { Non- } \\
\text { formatio } \\
\mathbf{n}\end{array}$ & $\begin{array}{c}\text { Inclusio } \\
\mathbf{n}\end{array}$ \\
\hline $\begin{array}{c}\text { Occurrences } \\
\text { of defects } \\
\%\end{array}$ & 8 & 6 & 3 & 3 \\
$\begin{array}{c}\text { Occurrences } \\
\text { of defects } \\
\%\end{array}$ & 40 & 30 & 15 & 15 \\
$\begin{array}{c}\text { Cumulative } \\
\text { Occurrence } \\
\text { s of defects }\end{array}$ & $\mathbf{4 0}$ & $\mathbf{7 0}$ & $\mathbf{8 5}$ & $\mathbf{1 0 0}$ \\
\hline
\end{tabular}

Based on data analysis, shrinkage had occurred eight times, micro shrinkage had occurred six times, inclusion \& non-formation had occurred three times in the batch tested. Since casting acceptance is based on elimination of all these defects, all of them are considered vital and the sequence of defect elimination was on the basis of number of occurrences of the defect (Shrinkage, Micro Shrinkage, Inclusion and Non-Formation).

\section{RESULT ANALYSIS}

Table-7. Defect check sheet (Batch Quantity: 2 Nos)

\begin{tabular}{|c|c|c|c|c|c|c|}
\hline Sl.no. & Melt No. & Stage of Rejection & \multicolumn{4}{|c|}{ Reas on for Rejection } \\
\hline & & & $\mathrm{s}$ & MS & I & $\mathrm{N}-\mathrm{F}$ \\
\hline 1 & $\begin{array}{c}\text { MG02 AP } \\
06\end{array}$ & $\begin{array}{l}\text { Floor, Radiography } \\
\text { Inspection }\end{array}$ & $\mathrm{Y}$ & - & - & $\mathrm{Y}$ \\
\hline 2 & $\begin{array}{c}\mathrm{M} 502 \mathrm{AP} \\
15\end{array}$ & Radiography Inspection & $\mathrm{Y}$ & $\mathrm{Y}$ & - & - \\
\hline 3 & $\begin{array}{c}\mathrm{MG} 02 \mathrm{AP} \\
17\end{array}$ & Radiography Inspection & $\mathrm{Y}$ & $\mathrm{Y}$ & $\mathrm{Y}$ & - \\
\hline 4 & $\begin{array}{l}\mathrm{MG} 02 \pi \mathrm{U} \\
08\end{array}$ & Radiography Inspection & $\mathrm{Y}$ & $\mathrm{Y}$ & $\mathrm{Y}$ & - \\
\hline 5 & $\begin{array}{l}\text { MG } 02 \\
\text { NR } 40\end{array}$ & $\begin{array}{c}\text { Floor, Radiography } \\
\text { Inspection }\end{array}$ & $\mathrm{Y}$ & - & - & $\mathrm{Y}$ \\
\hline 6 & $\begin{array}{l}\text { MG } 02 \\
\text { DR } 05\end{array}$ & Radiography Inspection & $\mathrm{Y}$ & $\mathrm{Y}$ & - & - \\
\hline 7 & $\begin{array}{c}\mathrm{MG} 02 \mathrm{U} \\
18\end{array}$ & $\begin{array}{l}\text { Floor, Radiography } \\
\text { Inspection }\end{array}$ & - & - & - & $\mathrm{Y}$ \\
\hline 8 & $\begin{array}{l}\mathrm{MG} 02 \mathrm{U} \\
24\end{array}$ & Radiography Inspection & - & $\mathrm{Y}$ & $\mathrm{Y}$ & - \\
\hline 9 & $\underset{33}{\mathrm{MG} 02 \mathrm{U}}$ & Radiography Inspection & $\mathrm{Y}$ & $\mathrm{Y}$ & - & - \\
\hline 10 & $\begin{array}{l}\mathrm{MG} 02 \pi \\
40\end{array}$ & Radiography Inspection & $\mathrm{Y}$ & - & - & - \\
\hline
\end{tabular}




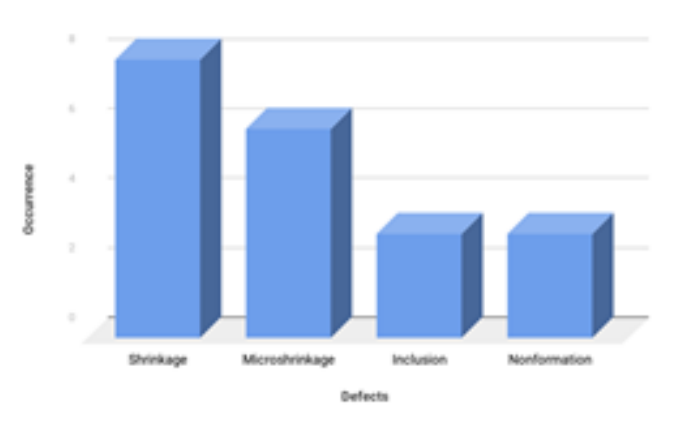

Fig.2. Graph for No. of Occurrences vs Defect.

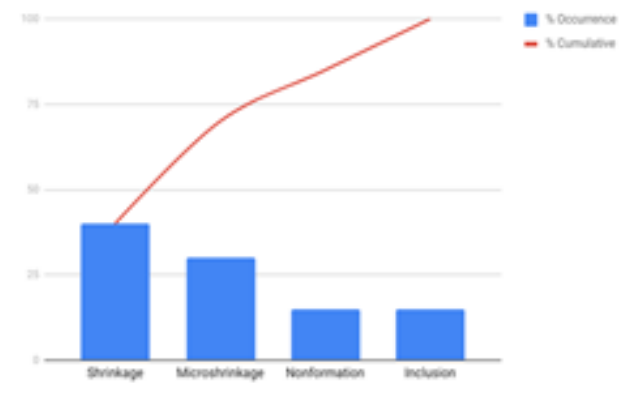

Fig. 3. Cumulative occurrence of defects

\subsection{Foreseeing probable resistance}

Deming wheel tool used to identify the solution and selection of feasible solution.

\subsubsection{Reasons for resistance}

1. Cavities for multi layered mesh \& reservoir needs to be hand cut

2. Difficulty in locating the shaped ingates during mould preparation

3. Difficulty in locating reservoir during mould making

\subsubsection{Overcome by}

1. Using thermocol to provide cavities for multi layered mesh \& reservoir required were provided with pins.

\section{TRIAL IMPLEMENTATION}

While implementing the proposed modifications step by step, the following results were recorded from the trials:

\subsection{Trial -1}

1. Introduction of riser

2. Reduction of pouring temperature from $750 \mathrm{C}$ to $740 \mathrm{C}$

3. Introduction of chills at the thick portion

\subsubsection{Trial-1 Result}

1. Shrinkage and Micro Shrinkage persisted. Hence the casting was rejected

\subsection{Trial -2}

1. Pouring temperature was further reduced from $740 \mathrm{C}$ to $730 \mathrm{C}$

2. Introduction of Zircon Sand for Facing during mould making.

\subsubsection{Trial - 2 Result}

1. Shrinkage and Micro Shrinkage were completely avoided. However, Inclusion and Non - Formation was noticed and hence the casting was rejected
2. Locations where shaped ingates \& reservoirs where

\subsection{Trial -3}

1. Changing of Gating Ratio from 2.8:3.6:1 to 3.7:4.7:1

2. Changing the location of Ingate

\subsubsection{Trial-3 Result}

1. Non-Formation was avoided but inclusions were noticed. Hence the casting was rejected

5.4 Trial -4

1. Multi-layer mesh $(3 \times 2 \times 9)$ were introduced.

2. Flow offs were introduced at Runner bar end.

\subsubsection{Trial - 4 Result}

1. All defects encountered were successfully eliminated and $100 \%$ Radiographic Quality Castings were produced.

\section{REGULAR IMPLEMENTATION}

Table 8. Data on regular implementation.

\begin{tabular}{|c|c|c|c|c|c|c|}
\hline $\begin{array}{l}\text { Sl. } \\
\text { no }\end{array}$ & $\begin{array}{l}\text { Melt } \\
\text { No. }\end{array}$ & Shrinkage & $\begin{array}{l}\text { Micro } \\
\text { Shrinka } \\
\text { ge }\end{array}$ & Non-Formation & Inclusion & Remarks \\
\hline 1 & $\begin{array}{l}\text { MG 04 } \\
\text { JU 46 }\end{array}$ & NIL & NIL & NIL & NIL & ACCEPTED \\
\hline 2 & $\begin{array}{l}\text { MG } 04 \\
\text { JU } 53\end{array}$ & NIL & NIL & NIL & NIL & ACCEPTED \\
\hline 3 & $\begin{array}{l}\text { MG } 04 \\
\text { JU } 64\end{array}$ & NIL & NIL & NIL & NIL & ACCEPTED \\
\hline 4 & $\begin{array}{l}\text { MG } 04 \\
\mathrm{JU} 72\end{array}$ & NIL & NIL & NIL & NIL & ACCEPTED \\
\hline 5 & $\begin{array}{l}\text { MG 04 } \\
\text { JY 04 }\end{array}$ & NIL & NIL & NIL & NIL & ACCEPTED \\
\hline 6 & $\begin{array}{l}\text { MG 04 } \\
\text { JY } 18\end{array}$ & NIL & NIL & NIL & NIL & ACCEPTED \\
\hline 7 & $\begin{array}{l}\text { MG 04 } \\
\text { JY } 26\end{array}$ & NIL & NIL & NIL & NIL & ACCEPTED \\
\hline 8 & $\begin{array}{l}\text { MG } 04 \\
\text { JY } 37\end{array}$ & NIL & NIL & NIL & NIL & ACCEPTED \\
\hline 9 & $\begin{array}{l}\text { MG 04 } \\
\text { JY 48 }\end{array}$ & NIL & NIL & NIL & NIL & ACCEPTED \\
\hline 10 & $\begin{array}{l}\text { MG } 04 \\
\text { JY } 60\end{array}$ & NIL & NIL & NIL & NIL & ACCEPTED \\
\hline 11 & $\begin{array}{l}\text { MG 04 } \\
\text { JY } 68\end{array}$ & NIL & NIL & NIL & NIL & ACCEPTED \\
\hline 12 & $\begin{array}{l}\text { MG } 04 \\
\text { AU } 20\end{array}$ & NIL & NIL & NIL & NIL & ACCEPTED \\
\hline 13 & $\begin{array}{l}\text { MG } 04 \\
\text { JY } 51\end{array}$ & NIL & NIL & NIL & NIL & ACCEPTED \\
\hline 14 & $\begin{array}{l}\text { MG } 04 \\
\text { AU } 60\end{array}$ & NIL & NIL & NIL & NIL & ACCEPTED \\
\hline 15 & $\begin{array}{l}\text { MG 04 } \\
\text { SR } 14\end{array}$ & NIL & NIL & NIL & NIL & ACCEPTED \\
\hline 16 & $\begin{array}{l}\text { MG 04 } \\
\text { SR 26 }\end{array}$ & NIL & NIL & NIL & NIL & ACCEPTED \\
\hline 17 & $\begin{array}{l}\text { MG } 04 \\
\text { NR } 10\end{array}$ & NIL & NIL & NIL & NIL & ACCEPTED \\
\hline 18 & $\begin{array}{l}\text { MG 04 } \\
\text { NR 28 }\end{array}$ & NIL & NIL & NIL & NIL & ACCEPTED \\
\hline
\end{tabular}




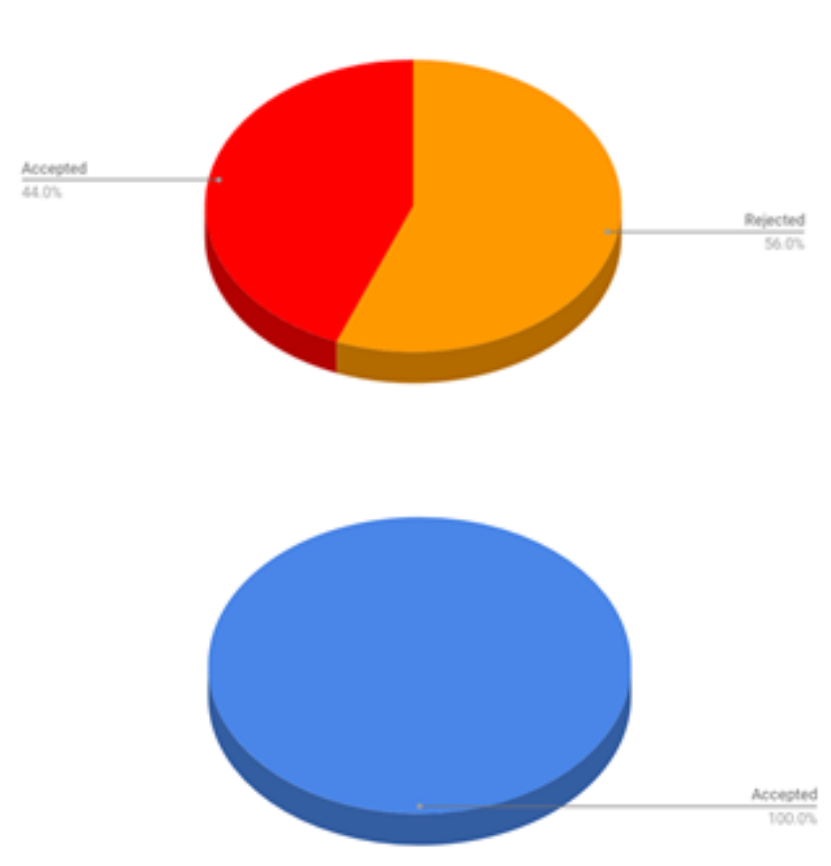

Fig. 4. (a) Old method; (b)New method

Table 9. Comparison of Old and New methods on regular implementation.

\begin{tabular}{ccc}
\hline Method & Old Method & New Method \\
\hline $\begin{array}{c}\text { No. of } \\
\text { poured }\end{array}$ & 36 & 36 \\
No. of \\
$\begin{array}{c}\text { Castings } \\
\text { accepted }\end{array}$ \\
$\begin{array}{c}\text { No. of } \\
\text { Castings } \\
\text { rejected }\end{array}$ \\
$\begin{array}{c}\text { \% of } \\
\text { Rejection }\end{array}$ \\
\hline
\end{tabular}

\section{CONCLUSION}

On regular implementation of the new method, Rs. $19,525 /$ - was saved per casting, which produced a total tangible benefit of Rs. 39,05,000/- for a batch of 200 castings. Additionally, there was increased work satisfaction on achievement, increased self-reliance in using complicated technologies and improved morale among employees.

\section{REFERENCES}

1. Lawler III, E.E., 1994. Total quality management and employee involvement: are they compatible?. Academy of Management Perspectives, 8(1), pp.68-76.

2. Blaga, P. and Jozsef, B., 2014. Human resources, quality circles and innovation. Procedia Economics and Finance, 15, pp.1458-1462.

3. Hill, S., 1991. Why quality circles failed but total quality management might succeed. British journal of industrial relations, 29(4), pp.541-568.

4. Kanji, G.K., 1990. Total quality management: the second industrial revolution. Total quality management, 1(1), pp.3-12.
5. Blackburn, R. and Rosen, B., 1993. Total quality and human resources management: lessons learned from Baldrige Award-winning companies. Academy of Management Perspectives, 7(3), pp.49-66.

6. Albers Mohrman, S., Tenkasi, R.V., Lawler, E.E. and Ledford, G.E., 1995. Total quality management: practice and outcomes in the largest US firms. Employee relations, 17(3), pp.26-41.

7. Duran, C., Çetindere, A. and Şahan, Ö., 2014. An analysis on the relationship between total quality management practices and knowledge management: The case of Eskişehir. Procedia-Social and Behavioral Sciences, 109, pp.65-77.

8. Hosseinabadi, R., Karampourian, A., Beiranvand, S. and Pournia, Y., 2013. The effect of quality circles on job satisfaction and quality of work-life of staff in emergency medical services. International emergency nursing, 21(4), pp.264-270.

9. Samson, D. and Terziovski, M., 1999. The relationship between total quality management practices and operational performance. Journal of operations management, 17(4), pp.393-409.

10. Anil, A.P. and Satish, K.P., 2016. Investigating the relationship between TQM practices and firm's performance: A conceptual framework for Indian organizations. Procedia Technology, 24, pp.554-561.

11. Sadikoglu, E. and Zehir, C., 2010. Investigating the effects of innovation and employee performance on the relationship between total quality management practices and firm performance: An empirical study of Turkish firms. International journal of production economics, 127(1), pp.13-26.

12. Wilkinson, A., Marchington, M., Goodman, J. and Ackers, P., 1992. Total quality management and employee involvement. Human Resource Management Journal, 2(4), pp.1-20.

13. Sadikoglu, E. and Zehir, C., 2010. Investigating the effects of innovation and employee performance on the relationship between total quality management practices and firm performance: An empirical study of Turkish firms. International journal of production economics, 127(1), pp.13-26.

14. Temtime, Z.T. and Solomon, G.H., 2002. Total quality management and the planning behavior of SMEs in developing economies. The TQM magazine, 14(3), pp.181-191.

15. Corredor, P. and Goñi, S., 2011. TQM and performance: Is the relationship so obvious?. Journal of Business Research, 64(8), pp.830-838.

16. Powell, T.C., 1995. Total quality management as competitive advantage: a review and empirical study. Strategic management journal, 16(1), pp.15-37.

17. Sahooa, S. and Yadava, S., 2018. Total Quality Management in Indian Manufacturing SMEs. Procedia Manufacturing, 21, pp.541-548.

18. Bourke, J. and Roper, S., 2017. Innovation, quality management and learning: Short-term and longer-term effects. Research Policy, 46(8), pp.1505-1518.

19. Prajogo, D.I. and Hong, S.W., 2008. The effect of TQM on performance in $R \& D$ environments: A perspective from South Korean firms. Technovation, 28(12), pp.855-863.

20. Ooi, K.B., 2014. TQM: A facilitator to enhance knowledge management? A structural analysis. Expert Systems with Applications, 41(11), pp.5167-5179. 